\title{
Genetic and clinical analysis of epithelial ovarian cancer in Greek patients
}

\author{
Plevris $\mathrm{N}^{1,2}$, Pappa KI ${ }^{3,4}$, Tsironis $\mathrm{G}^{5}$, Liontos $\mathrm{M}^{5}$, Charvalos $\mathrm{E}^{2}$, Rodolakis $\mathrm{A}^{4}$, Vlachos $\mathrm{G}^{4}$, Dimopoulos MA ${ }^{6}$, Zagouri F $\mathrm{F}^{5}$, Apostolou $\mathrm{P}^{7}$, \\ Konstantopoulou $\mathrm{I}^{7}$, Yanoukakos $\mathrm{D}^{7}$ and Florentia Fostira ${ }^{7 *}$ \\ ${ }^{1}$ Faculty of Medicine, School of Health Sciences, National and Kapodistrian University of Athens, Athens, Greece \\ ${ }^{2}$ Department of Obstetrics and Gynecology, 'IASO' Hospital, Athens, Greece \\ ${ }^{3}$ Cell and Gene Therapy Laboratory, Biomedical Research Foundation, Academy of Athens (BRFAA), Athens, Greece \\ ${ }^{4} 1$ st Department of Obstetrics and Gynecology, University of Athens School of Medicine, Athens, Greece \\ ${ }^{5}$ Oncology Unit, Department of Clinical Therapeutics, Alexandra Hospital, National and Kapodistrian University of Athens, Athens, Greece \\ ${ }^{6}$ Plasma Cell Dyscrasias Unit, Department of Clinical Therapeutics, School of Medicine, General Alexandra Hospital, National and Kapodistrian University of \\ Athens, Athens, Greece \\ ${ }^{7}$ Molecular Diagnostics Laboratory, INRaSTES, National Center for Scientific Research "Demokritos”, Athens, Greece
}

\begin{abstract}
Ovarian cancer is the deadliest cancer of the reproductive system for women in Western societies. The recent implementation of next-generation sequencing (NGS) technologies in the clinical setting is progressively revealing new genetic variants that are involved in ovarian cancer pathogenesis and allows the identification of patient subgroups for which prevention strategies can be developed. Herein, a subset of Greek patients diagnosed with epithelial ovarian cancer were selected for genetic testing by NGS, followed by MLPA. Clinical data were collected from 245 patients, 109 of whom were eligible for inclusion in the study. Overall, $14.7 \%$ and $8.3 \%$ carried germline BRCA1 and BRCA2 pathogenic variants, respectively, while $3.7 \%$ carried a damaging variant in additional cancer predisposing genes namely, MSH6 and RAD51C. Patients who were diagnosed at an advanced stage, i.e. III-IV, had significantly shorter overall survival compared to patients with stage I-II cancer at diagnosis. Genetic status and other patients' parameters, evaluated in the study, seem to significantly affect overall survival and disease recurrence.

Although our study did not reach statistical significance on overall survival for patients carrying germline pathogenic variants, identification of such patients is of high importance, given the opportunity they have for targeted therapies. The important role of multi-gene panel testing in unraveling cancer predisposition applies to every ovarian cancer patient, even when personal or familial criteria do not strongly fit with hereditary syndromes.
\end{abstract}

\section{Introduction}

Ovarian cancer is one of the most common causes of cancer-related deaths for women in Western societies [1]. Early diagnosis is quite challenging, mainly due to the non-specificity of symptoms; thus, the majority of patients are diagnosed at an advanced stage of the disease, which is correlated with worse survival and high relapse rates [2,3].

Ovarian cancer ranks fifth in cancer deaths among women and is the most lethal cancer of the female reproductive system. Notably, there were nearly 300,000 new cases worldwide in 2018 [4,5], with the number of new ovarian cancer cases per year being variable between countries. The Russian Federation and United Kingdom have a higher general population ovarian cancer percentage, while China has a low incidence. Interestingly, France, Italy and Greece had the lowest incidence with 3.5-4.5 new cases diagnosed per 100.000 women [6].

The majority of ovarian cancer cases have no relevant family history [7]. Contrarily, $\sim 10-15 \%$ of epithelial ovarian cancer diagnoses can be attributed to hereditary, germline pathogenic variants in key genes $[8,9]$. Among these genetic abnormalities, pathogenic variants in $B R C A 1$ and BRCA2 genes are by far the most common [10,11]. Once these pathogenic variants have been detected in a patient, targeted genetic screening can be offered to blood relatives in order to identify family members who are carriers, enabling intensive and personalized surveillance with early intervention or even cancer prevention.

In addition to $B R C A 1 / 2$, pathogenic variants in several other tumor suppressor genes have been associated with hereditary ovarian cancers. Pathogenic variants in these genes confer variable cancer risks, according to which they are classified into three categories: high penetrant genes that are associated with a relative risk higher than 5 (BRCA genes), medium penetrant genes that have a relative cancer risk between 1.5 and 5 and low-penetrant genes that confer a risk below 1.5 $[12,13]$.

The recent development and implementation of next-generation sequencing (NGS) technologies have made it possible to simultaneously analyze multiple cancer-associated genes [14] and to identify novel

*Correspondence to: Florentia Fostira, INRaSTES, Molecular Diagnostics Laboratory, NCSR 'Demokritos' Patriarchou Grigoriou \&Neapoleos, 15341, Aghia Paraskevi, Athens, Greece, Tel: +30 2106503841, Fax: +30 2106503876, E-mail: florentia@rrp.demokritos.gr

Key words: ovarian cancer, hereditary cancer, ngs, brcal, brca2

Received: October 12, 2020; Accepted: October 22, 2020; Published: October 28,2020 
candidate genes (other than $B R C A 1 / 2$ ) that are associated with ovarian cancer predisposition. However, testing by NGS can result in a large number of variants with uncertain significance (VUS), whose cancer risk-association is not clear, yet. On the other hand, implementation of this technology can parallel analyze multiple genes and patients, while can further investigate the genetic etiology and/or deregulated pathways that are involved in hereditary ovarian syndromes. These are crucial for the future development of molecular diagnostic strategies and targeted therapeutic approaches, which would significantly improve the prognosis for patients found to be carriers.

Herein, we have assessed the prevalence of pathogenic variants in a number of known or suspected genes in an unselected cohort of patients in Greece who were diagnosed with ovarian cancer or peritoneal or fallopian tube carcinoma. Additionally, we have assessed the patients' overall survival based on their genetic test result, as well as their risk of recurrence.

\section{Materials and methods}

\section{Study population}

Patients included in the study have been treated at Alexandra Hospital in Athens. All patients have agreed in participating in the study and have signed informed consent prior to having their blood drawn. Detailed pedigrees were obtained through dedicated phone interviews. Information concerning the overall survival (OS) of patients was collected up until May 2020.

\section{Next-generation sequencing}

Genetic analysis was performed using the cancer gene panel, TrusightCancer Panel (Illumina, San Diego, USA). Germline DNA was enzymatically fragmented, adaptor tagged, indexed and captured to target genomic regions of 42 cancer predisposing genes, following the manufacturer's instructions (Illumina, San Diego, USA).

Amplified libraries were evaluated qualitatively and quantitatively using Fragment Analyzer (Advanced Analytical Technologies, Heidelberg, Germany). Indexed libraries were sequenced on MiSeq using the Standard V2 kit performing 150 base paired-end reads, while FASTQ, BAM and VCF files were generated through IlluminaMiSeq Reporter; annotation was performed against the human reference genome GRCh38 using VariantStudio V.3. All identified pathogenic and likely pathogenic variants were confirmed by Sanger sequencing.

Genes selected for this study represent target variants with high, medium and low susceptibility in ovarian cancer development and patients' prognosis $[15,16]$. More specifically, pathogenic/likely pathogenic variants in the following 42 genes were monitored: $A P C$, ATM, BAP1, BLM, BMPR1A, BRCA1, BRCA2, BRIP1, CDH1, CDKN2A, CHEK2, DICER1, FANCM, FH, FLCN, MEN1, MLH1, MSH2, MSH6, MUTYH, NBN, NF1, NF2, PALB2, PMS1, PMS2, PTEN, RAD51C, RAD51D, RB1, RECQL4, RET, SDHB, SDHC, SDHD, SLX4, SMAD4, STK11, TP53, TSC1, TSC2, VHL.

\section{Multiplex ligation-dependent probe amplification analysis (MLPA)}

Detection of LGRs was conducted using the SALSA P002-D1 BRCA1 and P045-C1 BRCA2 /CHEK2 probemixes, following manufacturer's instructions, while analysis was performed with the Coffalyser software (MRC Holland, Amsterdam, the Netherlands).

\section{Statistical analysis}

Data were expressed as mean \pm standard deviation (S.D.) or median (in case of violation of normality) for continuous variables and as percentages for categorical data. The Kolmogorov-Smirnov test was used for normality analysis of the parameters. Comparison between the categories of genetic results in relation to quantitative and qualitative variables was performed using the One-Way ANOVA model and Chisquare test respectively. Uni-factorial analysis was conducted by using the Independent samples t-test or the Mann-Whitney test in case of violation of normality and Fisher's exact test to analyze the relation between the relapse (no vs. yes) and the quantitative, qualitative demographic and clinical variables, respectively.

The multi-factorial binary logistic regression model with enter method was used to identify independent demographic and clinical predictors of the relapse (no vs yes). Kaplan-Meier, Log Rank test and simple or multiple Cox proportional hazards models were used to estimate, compare survival functions and explore independent demographic and clinical predictors of survival, respectively. All tests were two-sided, statistical significance was set at $\mathrm{p}<0.05$. All analyses were carried out using the statistical package SPSS ver. 21.00 (IBM Corporation, Somers, NY, USA).

\section{Ethical approval}

All patients signed a consent form and had a thorough genetic counseling session prior to testing where all their questions and concerns have been answered. Access to the multidisciplinary team data was granted by the scientific committee of Alexandra hospital.

\section{Results}

\section{Patient cohort}

Overall, data were available from 246 patients, 109 of whom were eventually included in the study. A total of 137 patients were excluded due to age (younger than 18 years old), non-epithelial histology or the absence of contact details. Age at diagnosis ranged from 34 to 81 years old and the median age at diagnosis was 58 years. All data are presented in Figure 1.

In our cohort, 100 patients had primary ovarian cancer, 6 had primary tubal cancer, while 3 had primary peritoneal cancer. Of the patients with primary ovarian cancer, 14 had synchronous endometrial cancer, while one also had cervical cancer. The clinical, histopathologic and surgical characteristics of the patients are outlined in Table 1.

\section{Prevalence of germline cancer-predisposing variants}

Germline testing was performed through next-generation sequencing experimentation, followed by MLPA, and revealed prevalence for pathogenic variants of $25.68 \%$ (28/109). Of these, $14.7 \%$ and $8.3 \%$ carried $B R C A 1$ and $B R C A 2$ pathogenic variants, respectively. Moreover, $3.7 \%$ of the patients tested, carried pathogenic variants in additional genes namely, RAD51C (2 patients) and MSH6(1 patient). All identified germline pathogenic variants are presented in Table 2, while their prevalence and distribution are illustrated in Figure 2. Notably, an ATM missense variant (c.1516G $>$ T) with unknown clinical significance was found in one of the patients tested. No statistical significance was obtained when correlating patients' mutation status to the adjusted parameters of our research. 


\section{Overall survival is negatively affected by late diagnosis}

Herein, we have assessed whether the carrier of a germline pathogenic variant has an impact on patients' OS at 48 and 60 months. Based on our results, the vast majority of our patients carried pathogenic variants in BRCA1 and BRCA2 genes, with only three patients carrying pathogenic variants in additional genes. In order to achieve unbiased results, we have based our statistical analysis solely on patients carrying $B R C A 1$ or $B R C A 2$ pathogenic variants.

Our analysis showed that non-carrier patients have not significantly shorter OS at 60 months, when compared to patients who carried $B R C A$ pathogenic variants (60 months' survival: negative, $52.4 \%$ (39-66\%), vs. positive, $63.4 \%$ (39-85\%; $\mathrm{p}=0.128)$. All results are illustrated in Figure 3. Interestingly, the overall survival of carriers was improved and reached statistical significance, when all pathogenic variants carriers were included in the analysis (HR: $2.36,95 \%$ CI 1,01-5.75; $\mathrm{p}=0.050$ ) (Supplementary Table 1).

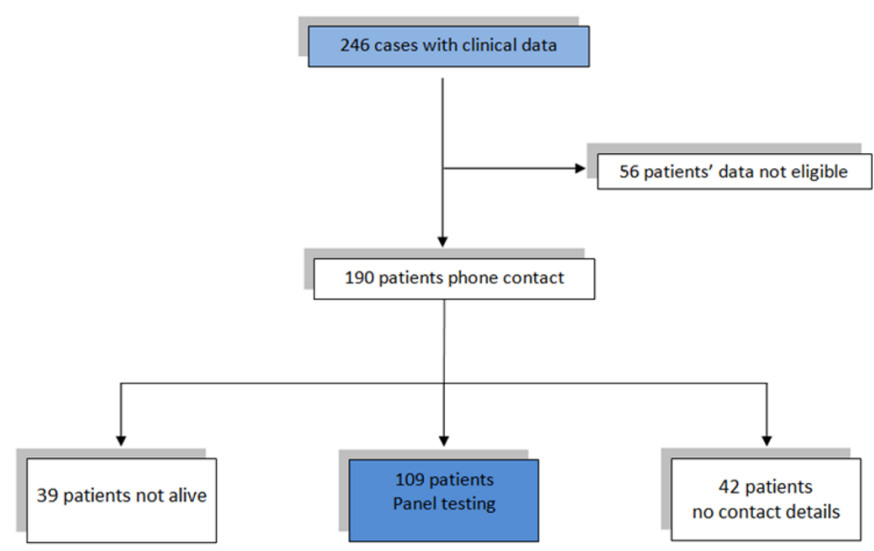

Figure 1. Study design outline

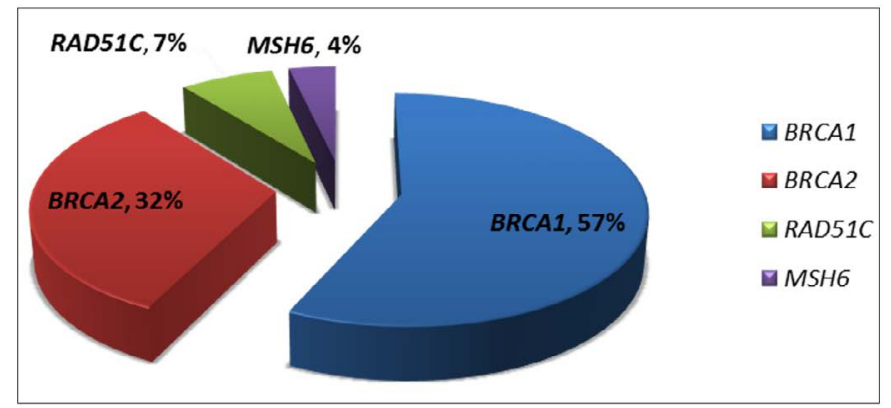

Figure 2. Prevalence and distribution of germline pathogenic variants in ovarian cancer predisposing genes

Table 1. Demographic, clinical and histopathological characteristics of the patient cohort

\begin{tabular}{|c|c|c|c|}
\hline \multirow{2}{*}{ Histological Subtype } & & $\mathbf{N}$ & $\mathbf{\%}$ \\
\hline \multirow{2}{*}{ Grade } & Serous & 98 & 89,9 \\
\cline { 2 - 4 } & EC/CC & 11 & 10,1 \\
\hline \multirow{2}{*}{ Stage } & Low & 6 & 5,5 \\
\hline \multirow{2}{*}{ Initial treatment } & High & 103 & 94,5 \\
\cline { 2 - 4 } & I-II & 19 & 17,4 \\
\hline \multirow{2}{*}{ Age } & III-IV & 90 & 82,6 \\
\hline & Surgical & 85 & 78,0 \\
\hline & & 24 & 22,0 \\
\hline
\end{tabular}

Table 2. Description of germline pathogenic variants among ovarian cancer patients

\begin{tabular}{|c|c|c|c|}
\hline Patient ID & Gene & DNA variation & Protein consequence \\
\hline F2656 & $B R C A 1$ & c. $3340 \mathrm{G}>\mathrm{T}$ & p.(Glu1114Ter) \\
\hline F2507 & $B R C A 1$ & c.3375_3376del & p.(Pro1126IlefsTer6) \\
\hline F0006 & $B R C A 1$ & c. $3700 \_3704 \mathrm{del}$ & p.(Val1234GlnfsTer8) \\
\hline F2071 & $B R C A 1$ & c. $3700 \_3704 \mathrm{del}$ & p.(Val1234GlnfsTer8) \\
\hline F2118 & $B R C A 1$ & c.3700_3704del & p.(Val1234GlnfsTer8) \\
\hline F2208 & $B R C A 1$ & c.3700_3704del & p.(Val1234GlnfsTer8) \\
\hline F3067 & $B R C A 1$ & c.3756_3759del & p.(Ser1253ArgfsTer10) \\
\hline F3108 & $B R C A 1$ & c.4964_4982del & p.(Ser1655TyrfsTer16) \\
\hline F1949 & $B R C A 1$ & c. $5212 \mathrm{G}>\mathrm{A}$ & p.(Gly1738Arg) \\
\hline F1895 & $B R C A 1$ & c. $5251 \mathrm{C}>\mathrm{T}$ & p.(Arg1751Ter) \\
\hline F2519a & $B R C A 1$ & c.5266dup & p.(Gln1756ProfsTer74) \\
\hline F2707 & $B R C A 1$ & c.5266dup & p.(Gln1756ProfsTer74) \\
\hline F3313 & $B R C A 1$ & c.5266dup & p.(Gln1756ProfsTer74) \\
\hline F2454 & $B R C A 1$ & c.5407-754_5592+8273del & p.(Gly1803_Tyr1863del) \\
\hline F3312 & $B R C A 1$ & c.5407-754_5592+8273del & p.(Gly1803_Tyr1863del) \\
\hline F0013 & $B R C A 1$ & c. $5467 \mathrm{G}>\mathrm{A}$ & p.Gly1803_Ala1813del \\
\hline F0023 & $B R C A 2$ & c. $2339 \mathrm{C}>\mathrm{G}$ & p.(Ser780Ter) \\
\hline F2975 & $B R C A 2$ & c.2644del & p.(Leu882PhefsTer13) \\
\hline F0025 & $B R C A 2$ & c.5576_5579del & p.(Ile1859LysfsTer3) \\
\hline F2483a & $B R C A 2$ & c. $7806-2 \mathrm{~A}>\mathrm{T}$ & p.Ala2603_Arg2659del \\
\hline F3136 & $B R C A 2$ & c. $7879 \mathrm{~A}>\mathrm{T}$ & p.(Ile2627Phe) \\
\hline F0017 & $B R C A 2$ & c.9016_9017del & p.(Tyr3006GlnfsTer11) \\
\hline F2093 & $B R C A 2$ & c.9089_9090insA & p.(Thr3033AsnfsTer11) \\
\hline F3187 & $B R C A 2$ & c. $9104 \mathrm{~A}>\mathrm{C}$ & p.(Tyr3035Ser) \\
\hline F0020 & $B R C A 2$ & c. $9117 \mathrm{G}>\mathrm{A}$ & p.Val2985GlyfsTer4 \\
\hline F3107 & MSH6 & c.776del & p.(Gly259ValfsTer20) \\
\hline F2269 & RAD51C & c. $706-2 A>G$ & p.Val236_Ala279del \\
\hline F3166 & $R A D 51 C$ & c. $706-2 \mathrm{~A}>\mathrm{G}$ & p.Val236_Ala279del \\
\hline
\end{tabular}

Variant nomenclature was based on the following reference sequences: $B R C A 1$ NM_007294.4; BRCA2 NM_000059.4; MSH6 NM_000179.3; RAD5IC NM_058216.3

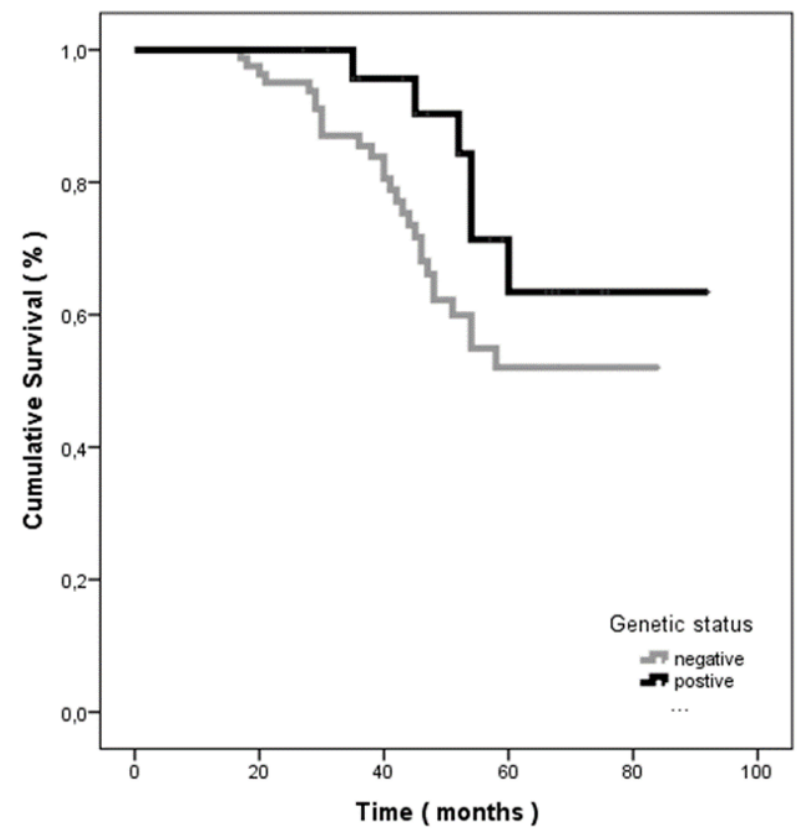

Figure 3. Sixty months' ovarian cancer specific survival according to genetic status of probands. Positive resembles to $B R C A 1$ and $B R C A 2$ pathogenic variant carriers and negative to panel negative probands, respectively 
In addition to that, patients diagnosed at a later stage, i.e. III-IV, had statistically significant shorter overall survival when compared to patients with a diagnosis of an earlier stage, i.e. I-II $(\mathrm{p}=0.017)$. A detailed graphical representation is depicted in Figure 4. Otherwise, overall survival was not significantly affected by laterality at diagnosis $(\mathrm{p}=0.273)$, initial treatment (surgery or neoadjuvant chemotherapy, $\mathrm{p}=0.500)$, histological subtype $(\mathrm{p}=0.655)$ or age at diagnosis $(\mathrm{p}=0.579)$. Median time to adjuvant chemotherapy was calculated as 34 days for patient treated with upfront cytoreductive surgery. All data are summarized in Table 3.

Using multiple Cox proportional hazards models, we observed that stage III-IV (HR: 7.65, 95\% CI 1.02-57.39; $\mathrm{p}=0.048$ ) significantly increases the hazard of death from ovarian cancer. No other tested factor seemed to significantly affect overall survival. Data are outlined in Table 4.

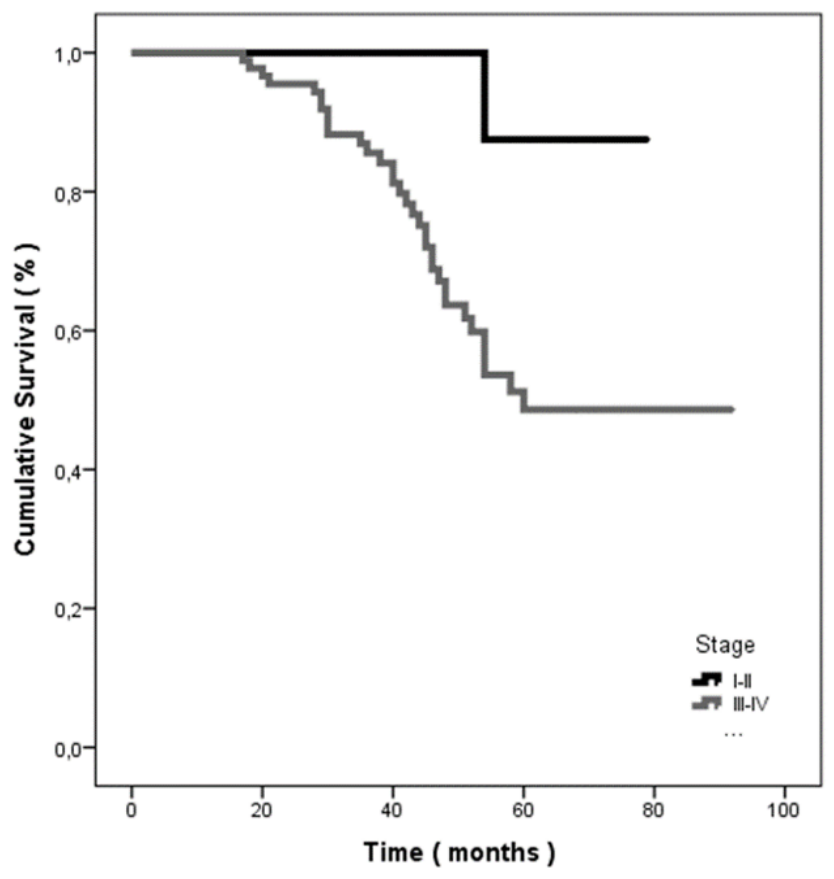

Figure 4. Sixty months' ovarian cancer specific survival defined by stage of diagnosis

Table 4. Multiple Cox proportional hazard regression analysis to overall survival including only $B R C A 1$ and $B R C A 2$ carriers

\begin{tabular}{|c|c|c|c|c|c|c|}
\hline & & $\mathbf{N}$ & $\begin{array}{l}\text { Survival } \\
60 \text { months }\end{array}$ & \multicolumn{2}{|c|}{$95 \% \mathrm{CI}$} & p-value \\
\hline \multirow{2}{*}{ Genetic result } & Negative result & 81 & $52,4 \%$ & $39 \%$ & $66 \%$ & \multirow{2}{*}{0.128} \\
\hline & Positive result & 25 & $63,4 \%$ & $39 \%$ & $85 \%$ & \\
\hline \multirow{2}{*}{ Laterality } & Bilateral & 79 & $50 \%$ & $38 \%$ & $64 \%$ & \multirow{2}{*}{0.273} \\
\hline & Unilateral & 27 & $73 \%$ & $48 \%$ & $98 \%$ & \\
\hline \multirow{2}{*}{ Stage } & I-II & 17 & $88 \%$ & $66 \%$ & $100 \%$ & \multirow{2}{*}{0.017} \\
\hline & III-IV & 89 & $48 \%$ & $34 \%$ & $62 \%$ & \\
\hline \multirow{3}{*}{ Initial treatment } & Surgical & 83 & $56 \%$ & $40 \%$ & $72 \%$ & \multirow{2}{*}{0.500} \\
\hline & NACT & 23 & $46 \%$ & $21 \%$ & $71 \%$ & \\
\hline & & $\mathrm{N}$ & Survival 48 months & \multicolumn{2}{|c|}{$95 \% \mathrm{CI}$} & p-value \\
\hline \multirow{3}{*}{ Histological subtype } & $\mathrm{EC} / \mathrm{CC}$ & 10 & $80 \%$ & $57 \%$ & $100 \%$ & \multirow{2}{*}{0.665} \\
\hline & Serous & 96 & $68 \%$ & $53 \%$ & $79 \%$ & \\
\hline & & & HR & \multicolumn{2}{|c|}{$95 \% \mathrm{CI}$} & p-value \\
\hline Age & & & 1.01 & 0.98 & 1.04 & 0.579 \\
\hline
\end{tabular}

Notably, the majority of patients in our cohort $(68.8 \%$; $75 / 109)$ were alive up until 60 months after their initial diagnosis. This result is distributed among the study groups as $74.42 \%$ of panel negative patients and $25.68 \%$ of patients carrying a pathogenic variant.

\section{Discussion}

Our study involves one of the first attempts to record and genetically assess ovarian cancer patients in Greece. Herein, a relatively large number of the tested patients, i.e. $25.6 \%$, have been identified to carry germline pathogenic variants in four genes, when compared to already published evidence. We have observed that late stage at ovarian cancer diagnosis can significantly reduce OS. On the contrary, we have not been able to show improved prognosis in BRCA1/2 carriers, as previously shown [17].

More specifically, previous studies have shown that ovarian cancer patients carrying $B R C A 1 / 2$ pathogenic variants have longer OS [18], mainly due to better responses to platinum-based chemotherapy [19]. In fact, it has been reported that $B R C A 1 / 2$ carrier status is an independent factor of improved survival for advanced stage ovarian cancer [20]. This can be explained by homologous recombination deficiency, which leads to reduced DNA-repairing capacity [21] and the inability to repair double-strand DNA breaks caused by platinum-based chemotherapy agents [22]. However, whether increased 5-year survival rates ultimately translate into long-term survival benefits remains unclear. Kotsopoulos et al. studied 1421 patients with ovarian cancer and found that although $B R C A$ carriers exhibited an initial survival advantage, which was not associated with a higher survival rate, when prolonged to a 10 -year follow-up [23]. Instead, the study highlighted the absence of residual disease at resection as the strongest predictor of long-term survival, as it has also been reported by Martín-Cameán et al. [24]. A possible explanation for not observing OS advantage in $B R C A$ carriers in our study, is the overall increased OS in our patient cohort, i.e. $68.8 \%$ being alive up until 60 months. Interestingly, data from the SEER database for years 2015-2019, suggest that over-all 5-year survival from ovarian cancer diagnosis was $47.6 \%$. Although we cannot precisely explain the longer survival in our study, we can speculate that meticulous initial surgical treatment could have possibly favored increased OS in our study population.

The debate regarding initial treatment remains heated between healthcare providers, but it is now widely accepted that complete cytoreduction remains the most important prognostic factor, whether it is offered upfront as primary debulking surgery (PDS) or as interval debulking surgery (IDS) after 3 or more cycles of chemotherapy $[25,26]$. Guidelines published by ASCO and SGO suggest that even women who have respectable peritoneal disease and are fit for surgery might be offered either PDS or NACT as initial treatment [27]. These recommendations are based on two prospective randomized studies the EORTC and Chorus trial - that have been criticized for the selection criteria of the patients enrolled and the surgical effort offered to patients measured by the amount of residual disease after surgical treatment. Complete cytoreduction for the CHORUS trial was around 30\% for patients undergoing both PDS and IDS, while in the EORTC trial the complete cytoreduction rate in the PDS group was only $19.4 \%[28,29]$.

Overall survival in our study was correlated with disease stage at presentation. In particular, patients at stage III-IV at diagnosis had statistically significantly shorter OS when compared to patients with stage I-II cancer at diagnosis. In line with our findings, there are data 
reporting that five-year survival remains poor for patients diagnosed at stage IV, reaching only $29.2 \%$, while patients diagnosed at stage I have a five-year survival rate of $92.4 \%$. Unfortunately, the percentage of patients diagnosed at each stage is usually $69 \%$ and $15 \%$, respectively [30].

Over the last two decades, a small improvement in mortality rates for patients with ovarian cancer has been observed, which can mostly be attributed to advances in surgical techniques and targeted therapy [31]. In this context, the implementation of gene panel testing in the clinical setting enables the identification of patients that can be benefited from targeted therapies, such as PARP inhibitors [32,33], but can also affect surgical interventions and radicality other than oncologist decisionmaking processes [34]. The ideal time for performing genetic testing for ovarian cancer is at the time of diagnosis. ASCO guidelines clearly state that patients receiving a negative germline genetic test result should pursue tumor testing, since $5-7 \%$ of patients might carry somatic variants [35]. In the meantime, during the challenging process of ovarian cancer prevention and treatment, genetic information should be distributed to all blood relatives, who can be significantly benefited in terms of prevention.

\section{Study's limitations}

The limitations of this study involve the patient enrolment at a single institution, so the socioeconomic status and origins of the patients are similar, while all surgical interventions were performed by the same surgical treatment team. In addition to that, the number of patients included in the study is quite limited to reach statistical significance, while the follow-up of the patients was limited to five years. Finally, we have not collected details on the chemotherapeutic treatments used for each patient, which can possibly affect patient survival.

\section{Conflict of interest}

The authors declare that there is no conflict of interest.

\section{Acknowledgements}

The authors would like to thank Rubina Novelli for the editing assistance and Antonios Galanos for the statistical analysis.

\section{References}

1. Lheureux S, Braunstein M, Oza AM (2019) Epithelial ovarian cancer: Evolution of management in the era of precision medicine. CA Cancer J Clin 69: 280-304. [Crossref]

2. Flaum N, Crosbie EJ, Edmondson RJ, Smith MJ, Evans DG (2020) Epithelial ovarian cancer risk: A review of the current genetic landscape. Clin Genet 97: 54-63. [Crossref]

3. Bhoola S, Hoskins WJ (2006) Diagnosis and management of epithelial ovarian cancer. Obstet Gynecol 107: 1399-410.

4. World cancer research fund (2018) Ovarian cancer statistics [Available from: https:// www.wcrf.org/dietandcancer/cancer-trends/ovarian-cancer-statistics].

5. Institute $\mathrm{NC}$ (2018) Cancer stat facts: Ovarian cancer.

6. Malvezzi M, Carioli G, Rodriguez T, Negri E, La Vecchia C (2016) Global trends and predictions in ovarian cancer mortality. Ann Oncol 27: 2017-2025.

7. Prat J, Ribe A, Gallardo A (2005) Hereditary ovarian cancer. Hum Pathol 36: 861-870.

8. Walsh T, Casadei S, Lee MK, Pennil CC, Nord AS (2011) Mutations in 12 genes for inherited ovarian, fallopian tube, and peritoneal carcinoma identified by massively parallel sequencing. Proc Natl Acad Sci U S A 108: 18032-18037.

9. Pennington KP, Walsh T, Harrell MI, Lee MK, Pennil CC, et al. (2014) Germline and somatic mutations in homologous recombination genes predict platinum response and survival in ovarian, fallopian tube, and peritoneal carcinomas. Clin Cancer Res 20: 764-775.
10. Ford D, Easton DF, Bishop DT, Narod SA, Goldgar DE (1994) Risks of cancer in BRCA1-mutation carriers. Breast Cancer Linkage Consortium. Lancet 343: 692-695.

11. Breast Cancer Linkage C (1999) Cancer risks in BRCA2 mutation carriers. J Natl Cancer Inst 91: 1310-1316.

12. Economopoulou P, Dimitriadis G, Psyrri A (2015) Beyond BRCA: new hereditary breast cancer susceptibility genes. Cancer Treat Rev 41: 1-8.

13. Easton DF, Pharoah PD, Antoniou AC, Tischkowitz M, Tavtigian SV, et al. (2015) Gene-panel sequencing and the prediction of breast-cancer risk. N Engl J Med 372: 2243-2257.

14. Toss A, Tomasello C, Razzaboni E, Contu G, Grandi G, et al. (2015) Hereditary ovarian cancer: not only BRCA 1 and 2 genes. Biomed Res Int 341723.

15. Castera L, Krieger S, Rousselin A, Legros A, Baumann JJ, et al. (2014) Next-generation sequencing for the diagnosis of hereditary breast and ovarian cancer using genomic capture targeting multiple candidate genes. Eur J Hum Genet 22: 1305-13.

16. Prapa M, Solomons J, Tischkowitz M (2017) The use of panel testing in familial breast and ovarian cancer. Clin Med (Lond) 17: 568-572.

17. Hoskins PJ, Gotlieb WH (2017) Missed therapeutic and prevention opportunities in women with BRCA-mutated epithelial ovarian cancer and their families due to low referral rates for genetic counseling and BRCA testing: A review of the literature. $C A$ Cancer J Clin 67: 493-506.

18. Huang YW (2018) Association of BRCA1/2 mutations with ovarian cancer prognosis An updated meta-analysis. Medicine (Baltimore) 97: e9380.

19. Alsop K, Fereday S, Meldrum C, deFazio A, Emmanuel C, et al. (2012) BRCA mutation frequency and patterns of treatment response in BRCA mutation-positive women with ovarian cancer: a report from the Australian Ovarian Cancer Study Group. J Clin Oncol 30: 2654-2663. [Crossref]

20. Gallagher DJ, Konner JA, Bell-McGuinn KM, Bhatia J, Sabbatini P, et al. (2011) Survival in epithelial ovarian cancer: a multivariate analysis incorporating BRCA mutation status and platinum sensitivity. Ann Oncol 22: 1127-1132.

21. Muggia F, Safra T (2014) 'BRCAness' and its implications for platinum action in gynecologic cancer. Anticancer Res 34: 551-556.

22. Foulkes WD (2006) BRCA1 and BRCA2: chemosensitivity, treatment outcomes and prognosis. Fam Cancer 5: 135-142.

23. Kotsopoulos J, Rosen B, Fan I, Moody J, McLaughlin JR, et al. (2016) Ten-year survival after epithelial ovarian cancer is not associated with BRCA mutation status. Gynecol Oncol 140: 42-47.

24. Martin-Camean M, Delgado-Sanchez E, Pinera A, Diestro MD, De Santiago J, et al (2016) The role of surgery in advanced epithelial ovarian cancer. Ecancermedicalscience 10: 666 .

25. du Bois A, Reuss A, Pujade-Lauraine E, Harter P, Ray-Coquard I (2009) Role of surgical outcome as prognostic factor in advanced epithelial ovarian cancer: a combined exploratory analysis of 3 prospectively randomized phase 3 multicenter trials: by the Arbeitsgemeinschaft Gynaekologische Onkologie Studiengruppe Ovarialkarzinom (AGO-OVAR) and the Groupe d'Investigateurs Nationaux Pour les Etudes des Cancers de l'Ovaire (GINECO). Cancer 115: 1234-144.

26. Chi DS, Eisenhauer EL, Zivanovic O, Sonoda Y, Abu-Rustum NR, et al. (2009) Improved progression-free and overall survival in advanced ovarian cancer as a result of a change in surgical paradigm. Gynecol Oncol 114: 26-31.

27. Wright AA, Bohlke K, Armstrong DK, Bookman MA, Cliby WA, et al. (2016) Neoadjuvant chemotherapy for newly diagnosed, advanced ovarian cancer: Society of gynecologic oncology and american society of clinical oncology clinical practice guideline. J Clin Oncol 34: 3460-3473.

28. Vergote I, Trope CG, Amant F, Kristensen GB, Ehlen T, et al. (2010) Neoadjuvant chemotherapy or primary surgery in stage IIIC or IV ovarian cancer. $N$ Engl $J$ Med 363: 943-953.

29. Kehoe S, Hook J, Nankivell M, Jayson GC, Kitchener H, et al. (2015) Primary chemotherapy versus primary surgery for newly diagnosed advanced ovarian cance (CHORUS): an open-label, randomised, controlled, non-inferiority trial. Lancet 386 249-257. [Crossref]

30. Matulonis UA, Sood AK, Fallowfield L, Howitt BE, Sehouli J (2016) Ovarian cancer Nat Rev Dis Primers 2: 16061.

31. Claussnitzer M, Cho JH, Collins R, Cox NJ, Dermitzakis ET, et al. (2020) A brief history of human disease genetics. Nature 577: 179-89. 
32. Federici G, Soddu S (2020) Variants of uncertain significance in the era of highthroughput genome sequencing: a lesson from breast and ovary cancers. $J$ Exp Clin Cancer Res 39: 46.

33. Robson ME, Bradbury AR, Arun B, Domchek SM, Ford JM, et al. (2015) American society of clinical oncology policy statement update: Genetic and Genomic testing for cancer susceptibility. J Clin Oncol 33: 3660-3667.
34. Konstantinopoulos PA, Norquist B, Lacchetti C, Armstrong D, Grisham RN, et al. (2020) Germline and somatic tumor testing in epithelial ovarian cancer: ASCO Guideline. J Clin Oncol 38: 1222-1245.

35. LaDuca H, Stuenkel AJ, Dolinsky JS, Keiles S, Tandy S, et al. (2014) Utilization of multigene panels in hereditary cancer predisposition testing: analysis of more than 2,000 patients. Genet Med 16: 830-837. [Crossref]

Copyright: $(2020$ Plevris N. This is an open-access article distributed under the terms of the Creative Commons Attribution License, which permits unrestricted use, distribution, and reproduction in any medium, provided the original author and source are credited. 